\title{
Impacts of psychological resilience on self-efficacy and quality of life in patients with diabetic foot ulcers: a prospective cross- sectional study
}

\author{
Dan Kuang ${ }^{1 \#}$, Dan-Feng Gu ${ }^{2 \#}$, Hong Cao ${ }^{1 \#}$, Qin-Fang Yuan ${ }^{2}$, Zhi-Xia Dong ${ }^{3}$, Dan Yu ${ }^{1}$, Xiao-Ming Shen ${ }^{3}$ \\ ${ }^{1}$ Department of Endocrinology, Affiliated Hospital of Jiangnan University, Wuxi, China; ${ }^{2}$ Nursing Department, Affiliated Hospital of Jiangnan \\ University, Wuxi, China; ${ }^{3}$ Department of Gastroenterological Surgery, Affiliated Hospital of Jiangnan University, Wuxi, China \\ Contributions: (I) Conception and design: D Kuang, DF Gu, H Cao, XM Shen; (II) Administrative support: XM Shen; (III) Provision of study \\ materials or patients: D Kuang, DF Gu, H Cao, QF Yuan, ZX Dong, D Yu; (IV) Collection and assembly of data: D Kuang, DF Gu, H Cao, QF \\ Yuan, ZX Dong, D Yu; (V) Data analysis and interpretation: D Kuang, DF Gu, H Cao; (VI) Manuscript writing: All authors; (VII) Final approval of \\ manuscript: All authors. \\ "These authors contributed equally to this work. \\ Correspondence to: Xiao-Ming Shen. Department of Gastroenterological Surgery, Affiliated Hospital of Jiangnan University, Hefeng Road, Wuxi \\ 214000, China. Email: sxm00211@aliyun.com.
}

Background: Patients with diabetic foot ulcer (DFU) usually have a poor quality of life (QoL) and selfefficacy, which is affected by many risk factors. However, the role of psychological resilience in QoL and selfefficacy in DFU patients has remained unclear.

Methods: This prospective cross-sectional study was performed in a single center from January 2018 to February 2020. A total of 98 DFU patients were enrolled in this study. Some demographic and clinical data were prospectively collected from participants. The psychological resilience of participants was assessed by Connor-Davidson resilience scale (CD-RISC). Self-efficacy was also assessed using the diabetes management self-efficacy scale (DMSES) and QoL was assessed by the 36-item short-form (SF-36) health survey. Univariable and multivariable linear regression were used to analyze the risk factors of self-efficacy and QoL. Then, logistic regression was used to analyze the predictors of psychological resilience among the participants.

Results: A CD-RISC score of more than 85 points was defined as high psychological resilience in this study; there were 28 participants diagnosed with high psychological resilience and 70 patients with low psychological resilience. Those with high psychological resilience had significantly higher selfefficacy, general health, vitality, social functioning, role emotional, and mental health than those with low psychological resilience. According to multivariable linear regression, low psychological resilience and older age were identified as risk factors of self-efficacy. On the contrary, low psychological resilience, older age, lower perceived social support and higher level of glycated hemoglobin were identified as risk factors of QoL. Finally, males had lower psychological resilience than females and those receiving more social support had higher psychological resilience than participants receiving less social support.

Conclusions: Some risk factors of QoL and self-efficacy were identified in this study and these results may provide some evidence for the improvement of QoL and self-efficacy in DFU patients. Being female and receiving higher social support were shown to have potential for improving psychological resilience in DFU patients.

Keywords! Psychological resilience; self-efficacy; quality of life (QoL); social support; diabetic foot ulcer (DFU)

Submitted Mar 31, 2021. Accepted for publication May 10, 2021.

doi: 10.21037/apm-21-967

View this article at: http://dx.doi.org/10.21037/apm-21-967 


\section{Introduction}

Diabetes mellitus (DM) has become the most important metabolic disease nowadays and there are over 400 million people with DM globally according to the report of the International Diabetes Federation in 2015 (1). It is expected that the number of diabetic patients will continue to increase over the coming decades (2). The blood sugar disorder of diabetic patients may induce injury of the blood vessels and peripheral nerves. Furthermore, advanced DM can cause some severe complications including diabetic nephropathy, diabetic retinopathy, and diabetic neuropathy (3). Diabetic foot ulcer (DFU) is another important complication of DM, which has attracted much attention from diabetic scholars.

The causes of DFU are both diabetic neuropathy and angiopathy. On the one hand, injury of blood vessels would lead to vascular stenosis, and then insufficient blood supply of the lower extremity. Lower limb ischemia then leads to limb ulcer and even gangrene (4). On the other hand, diabetic nephropathy inhibits patients from feeling pain or any discomfort, leaving feet vulnerable to being unwittingly squeezed and deformed (4). In a previous study, the incidence of DFU in diabetic patients older than 50 years was $8.1 \%$ (5), which was slightly higher than the average worldwide incidence (6). Patients with DFU usually have poor prognosis, and there is a diabetic amputation every 20 seconds (7). The annual mortality of DFU patients is as high as $11 \%$, and the mortality of amputation patients has reached $22 \%$ (8). In addition, the recurrence of DFU after healing is relatively high at up to over 50\% (9).

Poor quality of life (QoL) is also associated with DFU, especially when it recurs or deteriorates. In attempts to improve the QoL of DFU patients, many studies have been performed to analyze the risk factors of poor QoL and provide guidance for the diagnosis, treatment, and nursing of DFU. Alrub et al. reported that female gender and obesity were related to poorer QoL in Jordanian DFU patients (10). Al Ayed et al. reported that, in addition to gender and obesity, education, occupation, income, and the number of complications were predictors of poor QoL in DFU patients (11). A recent meta-analysis indicated that some other risk factors such as the level of C-reactive protein (CRP), ulcer size, and level of glycated hemoglobin (HbA1c) had significant impacts on the QoL of DFU patients (12). Increasingly, attention has been paid to the role of psychological factors in the QoL of DFU patients. Polikandrioti et al. reported that severe anxiety contributed to poor QoL in DFU patients (1). Psychological resilience refers to how people react psychologically and behaviorally to change in their external environment. It has been reported that improved psychological resilience was related to higher QoL in patients with inflammatory bowel disease, breast cancer, and hypertension (13-15). However, no related study has yet been performed to investigate the impacts of psychological resilience on QoL in DFU patients.

In this study, we prospectively enrolled DFU patients in our hospital to determine the role of psychological resilience in their QoL. Besides, self-efficacy represents the confidence of patients to complete certain activities independently and the ability to inject insulin facilitated an increased QoL among DM patients (16). We also analyzed the role of psychological resilience in regulating the selfefficacy of DFU patients, along with the risk factors of psychological resilience. We present the following article in accordance with the STROBE reporting checklist (available at http://dx.doi.org/10.21037/apm-21-967).

\section{Methods}

\section{Study design}

This was a prospective cross-sectional study conducted in a single center from January 2018 to February 2020. All procedures performed in this study involving human participants were in accordance with the Declaration of Helsinki (as revised in 2013). The study was approved by institutional ethics board of Affiliated Hospital of Jiangnan University (No.: 02017014) and informed consent was taken from all the patients.

\section{Study population}

The inclusion criteria of this study were as follows: patients who were diagnosed with DFU, older than 18 years, able to complete the questionnaires, and conscious to cooperate with the treatment. The exclusion criteria were as follows: patients who were diagnosed with other life-threatening diseases, had mental diseases, were participating in other clinical trials, and refused to complete the questionnaires.

\section{Data collection}

The following data were collected at the time of participant enrolment: gender, age, body mass index (BMI), marital 
status, educational status, occupational status, number of children, smoking history, perceived social support, treatment pattern, latest level of $\mathrm{HbA1c}$, classification of foot ulcer, psychological resilience, self-efficacy, and QoL scores.

\section{Measurements}

Social support as perceived by participants in this study was assessed by the multidimensional scale of perceived social support (MSPSS) questionnaire. The MSPSS questionnaire had been translated into Chinese and contained 12 items in total. Each item had a score from 1 point, representing 'very strongly disagree', to 7 points, representing 'very strongly agree'. The higher participants scored, the more social support they felt they had access to.

The classification of foot ulcer was based on the Wagner ulcer classification, which was divided into 5 grades including grade 1, superficial diabetic ulcer; grade 2, extended ulcer (to tendon, bone, or joint); grade 3, deep ulcer with abscess or osteomyelitis; grade 4, gangrene in a portion of the forefoot; and grade 5, extensive gangrene of the foot (17).

Psychological resilience of the participants was assessed by Connor-Davidson resilience scale (CD-RISC), which had been translated into Chinese and contained 25 items in total. Each item had a score from 0 point, representing 'never', to 4 points, representing 'always'. The total possible score of CD-RISC was 100 points and a score of more than 85 points defined high psychological resilience.

Self-efficacy was assessed using the diabetes management self-efficacy scale (DMSES). The DMSES had been translated into Chinese and contained a total of 20 items. Each item had a score from 0 points, representing 'cannot be completed at all', to 10 points, representing 'absolutely can be completed'. A higher score indicated higher participant self-efficacy.

The QoL of participants was assessed by the 36-item short-form health survey (SF-36). The SF-36 had been translated into Chinese and it contained 36 items in total covering 8 aspects of QoL. Each item had a different score and proportion. The higher participants scored, the better their QoL was.

\section{Statistical analysis}

All statistical analyses in this study were performed using SPSS 20.0 (IBM Corp., Armonk, NY, USA). Categorical variables were expressed as number and percentage and compared between 2 groups using chi-square test. Continuous variables were expressed as mean and standard deviation. Univariable and multivariable linear regression were used to analyze the risk factors of self-efficacy and QoL and determine the role of psychological resilience in regulating self-efficacy and QoL. Then, univariable and multivariable logistic regression were used to analyze the predictors of psychological resilience. A $\mathrm{P}$ value less than 0.05 represented statistical significance.

\section{Results}

We enrolled $98 \mathrm{DFU}$ patients in this study as shown in Table 1. A total of 68 of these participants were male, the mean age of the cohort was $67.3 \pm 13.5$ years, and their mean BMI was $24.7 \pm 7.9$. Marital status was recorded and 80 participants $(81.6 \%)$ were married. Regarding educational status, 37 participants $(37.8 \%)$ had received primary school education, 39 (39.8\%) had achieved middle school education, and 22 (22.4\%) had completed high school or above education. A total of 31 participants (31.6) were employed and 67 (68.4\%) were retired or unemployed. Most of these participants had at least 1 child and 44 (44.9\%) had at least 2 children. A total of 34 participants (34.7\%) had a history of smoking. According to MSPSS, the mean score of perceived social support was $68.9 \pm 10.4$ points. More than half $(57.1 \%)$ of the participants were undergoing insulin treatment, 20 (20.4\%) were taking oral hypoglycemic agents, and 22 (22.4\%) were receiving insulin combined with oral hypoglycemic agents. The mean level of HbA1c was $6.6 \% \pm 1.2 \%$. Based on CD-RISC, the mean score of psychological resilience in enrolled patients was $77.0 \pm 18.9$ points. The classification of foot ulcer in DFU patients is summarized in Table 2. A total of 30 participants $(30.6 \%)$ were diagnosed as grade $1,51(52.0 \%)$ were diagnosed as grade $2,11(11.2 \%)$ were diagnosed as grade $3,5(5.1 \%)$ were diagnosed as grade 4 , and $1(1.0 \%)$ was diagnosed as grade 5 .

A CD-RISC score of more than 85 points was defined as high psychological resilience. There were 28 participants with high psychological resilience and 70 with low psychological resilience in this study (Table 1). More participants with high psychological resilience were employed compared to those with low psychological resilience ( $50 \%$ vs. $24.3 \%, \mathrm{P}=0.013)$. Besides, patients with high psychological resilience received much more social support than those with low psychological resilience 
Table 1 Characteristics of enrolled DFU patients

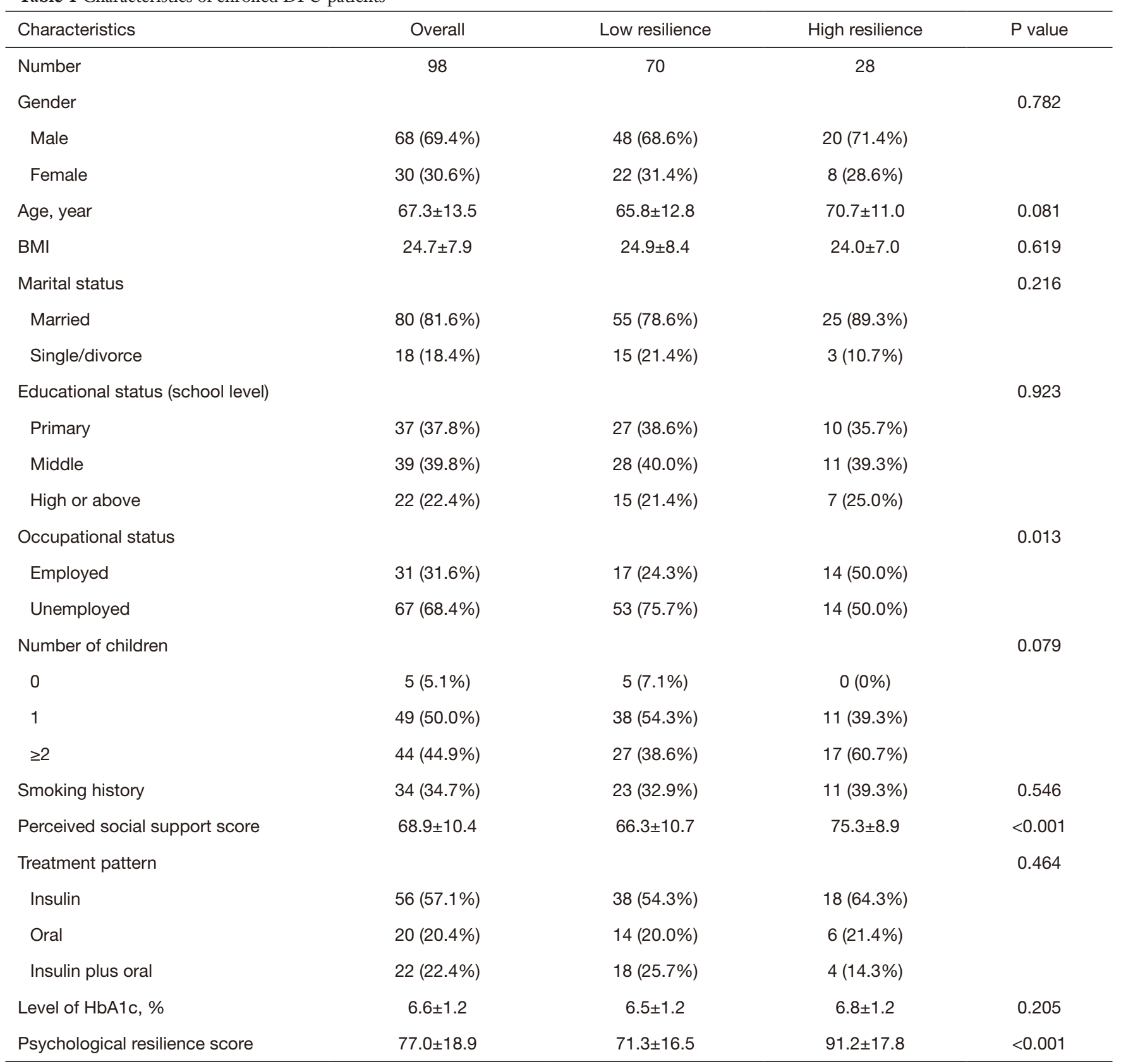

DFU, diabetic foot ulcer; BMI, body mass index; HbA1c, glycated hemoglobin.

(75.3 \pm 8.9 vs. $66.3 \pm 10.7, \mathrm{P}<0.001)$. More participants with high psychological resilience were diagnosed with grade 1 foot ulcer; however, no significant difference was found (Table 2). There was no significant difference in other variables between 2 groups.

The self-efficacy and QoL of DFU participants are summarized via DMSES and SF-36 scores in Table 3.
Participants with high psychological resilience had significantly higher levels of self-efficacy, general health, vitality, social functioning, role emotional, and mental health than participants with low psychological resilience (self-efficacy $67.0 \pm 7.9$ vs. $61.6 \pm 7.3, \mathrm{P}=0.003$; general health $60.0 \pm 9.3$ vs. $54.1 \pm 8.4, \mathrm{P}=0.005$; vitality $63.9 \pm 11.2$ vs. $53.1 \pm 11.3, \mathrm{P}<0.001$; social functioning $65.1 \pm 6.8$ vs. 
Table 2 Classification of foot ulcer in DFU patients

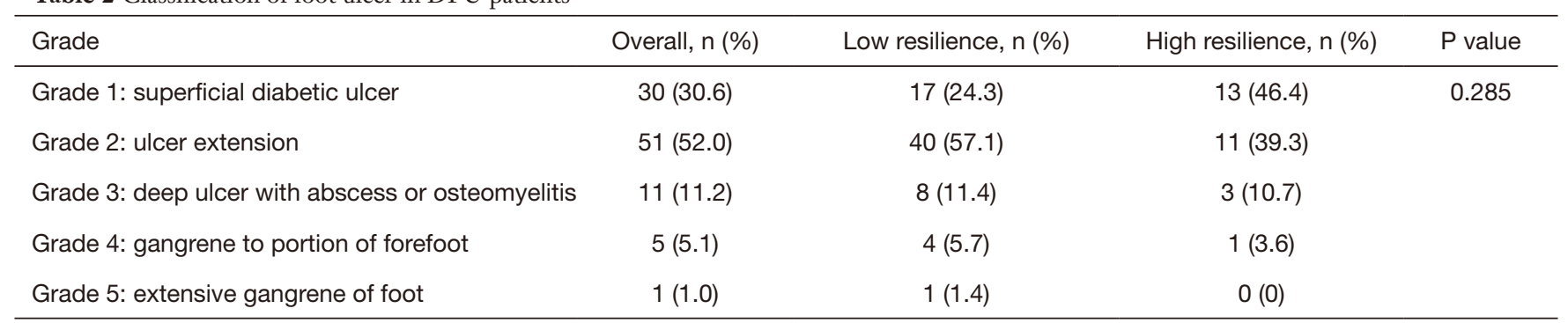

DFU, diabetic foot ulcer.

Table 3 Self-efficacy and QoL of enrolled DFU patients

\begin{tabular}{|c|c|c|c|c|}
\hline Variables & Overall & Low resilience & High resilience & $P$ value \\
\hline Physical functioning & $39.1 \pm 8.8$ & $38.6 \pm 8.1$ & $40.3 \pm 10.5$ & 0.399 \\
\hline Role physical & $24.0 \pm 6.0$ & $23.7 \pm 6.0$ & $24.9 \pm 6.1$ & 0.375 \\
\hline Bodily pain & $62.5 \pm 10.6$ & $61.9 \pm 10.2$ & $64.0 \pm 11.8$ & 0.412 \\
\hline Vitality & $55.9 \pm 12.0$ & $53.1 \pm 11.3$ & $63.9 \pm 11.2$ & $<0.001$ \\
\hline Social functioning & $59.2 \pm 7.0$ & $56.8 \pm 5.5$ & $65.1 \pm 6.8$ & $<0.001$ \\
\hline Role emotional & $36.6 \pm 8.4$ & $34.5 \pm 7.6$ & $41.5 \pm 8.3$ & $<0.001$ \\
\hline Mental health & $54.7 \pm 9.6$ & $51.9 \pm 8.3$ & $61.9 \pm 9.1$ & $<0.001$ \\
\hline
\end{tabular}

DFU, diabetic foot ulcer; QoL, quality of life.

Table 4 Univariable and multivariable linear regression analysis of risk factors of self-efficacy in DFU participants

\begin{tabular}{|c|c|c|c|c|}
\hline Variables & \multicolumn{2}{|c|}{ Univariable } & \multicolumn{2}{|l|}{ Multivariable } \\
\hline High psychological resilience & 5.264 (1.897 to 8.632$)$ & 0.003 & 4.340 (0.610 to 8.070$)$ & 0.023 \\
\hline Older age & $-0.288(-0.548$ to -0.028$)$ & 0.030 & $-0.081(-0.160$ to -0.002$)$ & 0.044 \\
\hline High education versus other & 0.086 (0.004 to 0.169$)$ & 0.041 & $0.078(-0.085$ to 0.241$)$ & 0.345 \\
\hline Higher level of & $-1.846(-3.180$ to -0.511$)$ & 0.007 & $-1.462(-2.764$ to 0.160$)$ & 0.068 \\
\hline
\end{tabular}

$\mathrm{Cl}$, confidence interval; HbA1c, glycated hemoglobin.

$56.8 \pm 5.5, \mathrm{P}<0.001$; role emotional $41.5 \pm 8.3$ vs. $34.5 \pm 7.6$, $\mathrm{P}<0.001$; mental health $61.9 \pm 9.1$ vs. $51.9 \pm 8.3, \mathrm{P}<0.001)$.

Risk factors of self-efficacy were analyzed using univariable and multivariable linear regression, as shown in Table 4. Low psychological resilience, older age, low education, unemployment, and higher level of HbAlc were identified as potential risk factors according to univariable linear regression. After adjusting by multivariable linear regression, low psychological resilience and older age were identified as risk factors of self-efficacy.

Then, risk factors of QoL in DFU participants were analyzed (Table 5). Low psychological resilience, older age, lower perceived social support, and higher level of HbA1c were identified as risk factors of QoL according to 
Table 5 Univariable and multivariable linear regression analysis of risk factors of QoL in DFU participants

\begin{tabular}{|c|c|c|c|c|}
\hline Variables & \multicolumn{2}{|l|}{ Univariable } & \multicolumn{2}{|l|}{ Multivariable } \\
\hline High psychological resilience & 5.943 (2.105 to 9.780$)$ & 0.003 & 5.647 (1.197 to 10.096$)$ & 0.013 \\
\hline Older age & $-0.191(-0.412$ to -0.015$)$ & 0.018 & $-0.295(-0.554$ to -0.036$)$ & 0.026 \\
\hline Married versus single/divorce & 6.465 (1.091 to 11.840$)$ & 0.019 & $3.664(-0.299$ to 7.628$)$ & 0.070 \\
\hline Higher perceived social support & $4.652(0.860$ to 8.443$)$ & 0.017 & $4.663(0.426$ to 8.900$)$ & 0.031 \\
\hline $\begin{array}{l}\text { Higher level of glycated } \\
\text { hemoglobin }\end{array}$ & $-0.102(-0.246$ to -0.018$)$ & 0.040 & $-0.266(-0.521$ to -0.011$)$ & 0.041 \\
\hline
\end{tabular}

DFU, diabetic foot ulcer; QoL, quality of life; $\mathrm{Cl}$, confidence interval.

Table 6 Univariable and multivariable logistic regression analysis of predictors of psychological resilience in enrolled DFU patients

\begin{tabular}{|c|c|c|c|c|}
\hline \multirow{2}{*}{ Variables } & \multicolumn{2}{|c|}{ Univariable } & \multicolumn{2}{|c|}{ Multivariable } \\
\hline & OR $(95 \% \mathrm{Cl})$ & $P$ value & OR $(95 \% \mathrm{Cl})$ & $P$ value \\
\hline Married vs. single/divorce & $2.542(1.107-5.838)$ & 0.028 & $2.388(0.986-5.248)$ & 0.060 \\
\hline High education vs. other & $1.874(1.042-3.426)$ & 0.042 & $1.656(0.906-3.021)$ & 0.101 \\
\hline
\end{tabular}

DFU, diabetic foot ulcer; OR, odds ratio; $\mathrm{Cl}$, confidence interval.

multivariable linear regression.

Predictors of psychological resilience were analyzed using univariable and multivariable logistic regression (Table 6). The results showed that males had lower psychological resilience than females and participants receiving more social support had higher psychological resilience than those receiving less social support.

\section{Discussion}

There were different grades of foot ulcer among the participants in our study, most of whom had grade 1 or grade 2 foot ulcers. Generally, most DFU participants were diagnosed with a grade of less than 4 according to a previous study $(1,18)$. However, conditions graded as such will still incur a significant decrease in the QoL of patients. Based on our results, the mean score of general health in all participants was $55.8 \pm 9.0$ out of 100 points, which was similar to previous reports $(19,20)$. Moreover, most participants in our study had low self-efficacy with a mean score of $63.2 \pm 7.9$ out of 200 points. Previous studies have also determined that self-efficacy may be significantly decreased in diabetic patients $(16,21)$. The ascertainment of risk factors of QoL and self-efficacy, therefore, has become an important topic to date.

In their study, Polikandrioti et al. found that living in a capital city and having high levels of anxiety was significantly harmful for patients' general health (1). Another study also found that female gender, overweight, peripheral vascular disease, and stress in life may be related to lower QoL in DFU patients (10). A study from Saudi Arabia found that many factors may affect the QoL of DFU patients, including age, educational status, occupational status, income, and complications (11). In the present study, low psychological resilience, older age, lower perceived social support, and higher level of HbAlc were identified as risk factors of QoL. The level of HbA1c reflected the adherence characteristics of DFU patients; adherence characteristics were verified in previous study as important factors of QoL in diabetic patients (22). Polikandrioti et al. also found that the decrease of social support was significantly related to the increase of depression in DFU 
patients and the presence of depression would further affect the QoL of patients (18). Most importantly, low psychological resilience was identified as an important risk factor of QoL in DFU participants in our study, which is similar to how it has been shown to influence patients with other diseases $(13,14)$.

Our study identified low psychological resilience and older age as risk factors of self-efficacy. Only a few studies have previously mentioned the risk factors of self-efficacy and a randomized controlled clinical trial confirmed that resilience training can improve the self-efficacy in diabetic patients (21). Some more well-designed studies are needed to further verify these findings.

Considering the important role of psychological resilience in QoL and self-efficacy in DFU patients, we also analyzed the predictors of psychological resilience in this study. Female gender and higher social support would strengthen psychological resilience of DFU patients according to our results. It is not difficult to understand the important role of social support in psychological resilience. Machisa et al. also concluded that increased social support was related to increased psychological resilience in survivors of intimate partner violence and patients with posttraumatic stress disorder $(23,24)$. However, the role of gender in regulating psychological resilience is not so clear. It may be explained by different socioeconomic status or physiological hormone levels of patients.

Some limitations existed in this study. Firstly, this prospective cross-sectional study enrolled only 98 DFU patients from a single center. The number of enrolled patients was relatively small and there may have been some resulting bias in the results. Secondly, some previous studies have also collected the adherence characteristics of DFU patients. These characteristics may be related to psychological resilience and affect the self-efficacy and QoL of patients. However, we did not collect these characteristics in this study, which may be improved in a future study. Thirdly, perceived social support, psychological resilience, self-efficacy, and QoL were assessed by several scales. In effect, some participants may not have filled out these scales truthfully, leading to the instability of the results.

Our study enrolled 98 DFU patients from a single center and found that most patients were classified as having either grade 1 or grade 2 of foot ulcer. According to linear regression analysis, low psychological resilience and older age were identified as risk factors of self-efficacy while low psychological resilience, older age, lower perceived social support, and higher level of HbA1c were identified as risk factors of QoL. These results may provide some evidence for the improvement of QoL and self-efficacy in DFU patients. Besides, female patients and receiving higher social support may help to improve psychological resilience in DFU patients.

\section{Acknowledgments}

Funding: This work was supported by the Scientific Research Foundation of Wuxi Health Commission (Q201929).

\section{Footnote}

Reporting Checklist: The authors have completed the STROBE reporting checklist. Available at http://dx.doi. org/10.21037/apm-21-967

Data Sharing Statement: Available at http://dx.doi. org/10.21037/apm-21-967

Conflicts of Interest: All authors have completed the ICMJE uniform disclosure form (available at http://dx.doi. org/10.21037/apm-21-967). The authors have no conflicts of interest to declare.

Ethical Statement: The authors are accountable for all aspects of the work in ensuring that questions related to the accuracy or integrity of any part of the work are appropriately investigated and resolved. All procedures performed in this study involving human participants were in accordance with the Declaration of Helsinki (as revised in 2013). The study was approved by institutional ethics board of Affiliated Hospital of Jiangnan University (No.: 02017014) and informed consent was taken from all the patients.

Open Access Statement: This is an Open Access article distributed in accordance with the Creative Commons Attribution-NonCommercial-NoDerivs 4.0 International License (CC BY-NC-ND 4.0), which permits the noncommercial replication and distribution of the article with the strict proviso that no changes or edits are made and the original work is properly cited (including links to both the formal publication through the relevant DOI and the license). 
See: https://creativecommons.org/licenses/by-nc-nd/4.0/.

\section{References}

1. Polikandrioti M, Vasilopoulos G, Koutelekos I, et al. Quality of Life in Diabetic Foot Ulcer: Associated Factors and the Impact of Anxiety/Depression and Adherence to Self-Care. Int J Low Extrem Wounds 2020;19:165-79.

2. Kateel R, Augustine AJ, Ullal S, et al. Development and validation of health related quality of life questionnaire (Indian scenario) in diabetic foot ulcer patients. Diabetes Metab Syndr 2017;11 Suppl 2:S651-S653.

3. Vileikyte L. Diabetic foot ulcers: a quality of life issue. Diabetes Metab Res Rev 2001;17:246-9.

4. Abdelbasset WK, Elsayed SH, Nambi G, et al. Potential efficacy of sensorimotor exercise program on pain, proprioception, mobility, and quality of life in diabetic patients with foot burns: A 12-week randomized control study. Burns 2021;47:587-93.

5. Jiang Y, Wang X, Xia L, et al. A cohort study of diabetic patients and diabetic foot ulceration patients in China. Wound Repair Regen 2015;23:222-30.

6. Steptoe A. Diabetes: Stress resilience and risk of type 2 diabetes mellitus. Nat Rev Endocrinol 2016;12:189-90.

7. Vadiveloo T, Jeffcoate W, Donnan PT, et al. Amputationfree survival in 17,353 people at high risk for foot ulceration in diabetes: a national observational study. Diabetologia 2018;61:2590-7.

8. Skrepnek GH, Mills JL Sr, Lavery LA, et al. Health Care Service and Outcomes Among an Estimated 6.7 Million Ambulatory Care Diabetic Foot Cases in the U.S. Diabetes Care 2017;40:936-42.

9. Dubský M, Jirkovská A, Bem R, et al. Risk factors for recurrence of diabetic foot ulcers: prospective followup analysis in the Eurodiale subgroup. Int Wound $\mathrm{J}$ 2013;10:555-61.

10. Alrub AA, Hyassat D, Khader YS, et al. Factors Associated with Health-Related Quality of Life among Jordanian Patients with Diabetic Foot Ulcer. J Diabetes Res 2019;2019:4706720.

11. Al Ayed M, Ababneh M, Alwin Robert A, et al. Factors Associated With Health-Related Quality of Life in Patients With Diabetic Foot Ulcer: A Cross-Sectional Study From Saudi Arabia. Cureus 2020;12:e8658.

12. Khunkaew S, Fernandez R, Sim J. Health-related quality of life among adults living with diabetic foot ulcers: a meta-analysis. Qual Life Res 2019;28:1413-27.
13. Sehgal P, Ungaro RC, Foltz C, et al. High Levels of Psychological Resilience Associated With Less Disease Activity, Better Quality of Life, and Fewer Surgeries in Inflammatory Bowel Disease. Inflamm Bowel Dis 2021;27:791-6.

14. Mohlin $\AA$, Axelsson U, Bendahl PO, et al. Psychological Resilience and Health-Related Quality of Life in Swedish Women with Newly Diagnosed Breast Cancer. Cancer Manag Res 2020;12:12041-51.

15. Qiu C, Shao D, Yao Y, et al. Self-management and psychological resilience moderate the relationships between symptoms and health-related quality of life among patients with hypertension in China. Qual Life Res 2019;28:2585-95.

16. Hsu HC, Chen SY, Lee YJ, et al. Pathways of diabetes distress, decisional balance, self-efficacy and resilience to quality of life in insulin-treated patients with type 2 diabetes: A 9-month prospective study. J Clin Nurs 2021;30:1070-8.

17. Wagner FW Jr. The diabetic foot. Orthopedics 1987;10:163-72.

18. Polikandrioti M, Vasilopoulos G, Koutelekos I, et al. Depression in diabetic foot ulcer: Associated factors and the impact of perceived social support and anxiety on depression. Int Wound J 2020;17:900-9.

19. Spanos K, Saleptsis V, Athanasoulas A, et al. Factors Associated With Ulcer Healing and Quality of Life in Patients With Diabetic Foot Ulcer. Angiology 2017;68:242-50.

20. Pedras S, Carvalho R, Pereira MG. Quality of Life in Portuguese Patients with Diabetic Foot Ulcer Before and After an Amputation Surgery. Int J Behav Med 2016;23:714-21.

21. Torabizadeh C, Asadabadi Poor Z, Shaygan M. The Effects of Resilience Training on the Self-Efficacy of Patients with Type 2 Diabetes: A Randomized Controlled Clinical Trial. Int J Community Based Nurs Midwifery 2019;7:211-21.

22. Jia J, Jenkins AJ, Quintiliani LM, et al. Resilience and diabetes self-management among African-American men receiving primary care at an urban safety-net hospital: a cross-sectional survey. Ethn Health 2020. doi: 10.1080/13557858.2020.1849566.

23. Machisa MT, Christofides N, Jewkes R. Social support factors associated with psychological resilience among women survivors of intimate partner violence in Gauteng, South Africa. Glob Health Action 2018;11:1491114. 
24. Imai R, Hori H, Itoh $M$, et al. Relationships of blood proinflammatory markers with psychological resilience and quality of life in civilian women with posttraumatic stress disorder. Sci Rep 2019;9:17905.

(English Language Editor: J. Jones)

Cite this article as: Kuang D, Gu DF, Cao H, Yuan QF, Dong ZX, Yu D, Shen XM. Impacts of psychological resilience on self-efficacy and quality of life in patients with diabetic foot ulcers: a prospective cross-sectional study. Ann Palliat Med 2021;10(5):5610-5618. doi: 10.21037/apm-21-967 